\title{
The Curative Effect of Traditional Chinese Medicine on Pediatric HFMD
}

\author{
Tian Jing, Wang Yanan, Liu Na \\ Shijiazhuang Medical College Hebei, Shijiazhuang, 050000
}

Keywords: Traditional Chinese medicine; Hand, foot and mouth disease in children; Therapeutic effect

\begin{abstract}
In order to explore the clinical effect of traditional Chinese medicine in the treatment of HFMD in children, a total of 120 HMFD children admitted to our hospital between December 2017 and March 2018 were randomly divided into a control group and an experimental group with 60 children in each group. The control group was addressed in the antiviral drug ribavirin, and the experimental group was treated with traditional Chinese medicine. After 7 days of treatment, clinical treatment effect and nursing satisfaction of the two groups were performed. Comparative analysis. The effective rate was $76.7 \%$ in the control group and $93.3 \%$ in the experimental group. The treatment efficiency and nursing satisfaction in the experimental group were significantly greater than those in the control group, $\mathrm{p}<0.05$ difference. Has statistical significance? The treatment of pediatric hand-foot-and-mouth disease in traditional Chinese medicine method can significantly improve the clinical treatment effect and nursing satisfaction of children, and it is worthy of promotion and application in clinical practice.
\end{abstract}

\section{Introduction}

HFMD is an acute infectious disease that is attributable to a variety of enteroviruses. Children under the age of four are high-risk groups. Hand-foot-mouth disease usually takes place in the late spring and early fall. If the child lives with hand, foot and mouth disease, it will show a series of clinical symptoms such as small herpes, ulcers, papules on the hands and feet, and papules into herpes. Afterwards, the child will have a fever, antioxidant and other symptoms [1]. Hand-footmouth disease does not have a corresponding cause in traditional Chinese medicine, but according to its clinical manifestations, HMFD can be attributed to diseases of wind and wet temperatures. Hand-foot-mouth disease has the characteristics of rapid changes in clinical conditions, so it should be discovered as soon as possible and take treatment, which has crucial significance for the rehabilitation of children. This article uses Chinese medicine method to treat infantile hand. Foot and mouth disease to obtain a good clinical effect, and is reported as follows.

\section{Data and Methods}

120 children with HFMD who were admitted to our hospital between December 2017 and March 2018 were randomly divided into a control group and an experimental group with 60 patients in each group. 120 children with hand-foot-mouth disease selected in this study met the diagnostic criteria for HFMD. There were 28 male patients and 32 female patients in the control group. The age range was $2-5$ years old and the average age was $(2.7 \pm 0.6)$ years old. The experimental group included 31 male patients and 29 female patients. The age range was 2-4 years old and the average age was $2.3 \pm 0.3$ years old. The differences in gender, age, etc. between the two groups were not statistically significant and are comparable $(\mathrm{P}>0.05)$.

Control group: Children in the control group was treated with the antiviral drug Ribavirin and all of them were addressed in isolation. They were provided with a light and easily digestible diet, and should be paid with more time to rest and good skin and oral care. In the course of treatment, children with high fever could be provided with oral antipyretics, children with higher blood cell could be treated with antibiotic and ribavirin antiviral treatment. Dosage and methods used: Ribavirin 10 mg/Kg.D, administered by intravenous drips once a day for 7 days. 
Experimental group: Children in the experimental group was treated with traditional Chinese medicine. In this study, Xiyanping injection and Pu Dilan oral liquid were utilized to treat children in this group. Dosage and methods: Inject 200-300ml of Xiyanping injection into 5\% glucose injection to infuse children once a day; let the children take Pudi Lan Oral Liquid 10ml/time, twice per day, the treatment course lasts for 7 days.

The control group was addressed in the antiviral drug ribavirin, and the experimental group was treated with traditional Chinese medicine. 7 days later, clinical treatment effect and nursing satisfaction of the two groups were performed with a comparative analysis. The clinical treatment effect was formulated according to the "criterion of clinical disease curative effect". Healing: The rash basically disappeared without a new recurrence. The child's body temperature and vital signs are natural, there were not any clinical symptoms of diarrhea and cough. The child's limbs are physically active with no neurological and psychiatric symptoms. The laboratory tests results of blood tests and blood biochemical tests are only natural. Effectivity: The symptoms of oral ulcers in children have been relieved, the rash on the trunk and the hand and foot areas has subsided, and other symptoms have also been improved. Invalidation: The dermatitis in the child's body and handfoot area was not significantly subsided, the child still had a fever, and symptoms of oral and skin infections [2].

All the data in this paper will be dealt with and analyzed by SPSS20.0 software. Measured data are expressed using mean value. $\mathrm{P}<0.05$ means discrepancy is statistically significant.

\section{Result}

Table 1. Comparison of overall treatment effect between two groups of patients

\begin{tabular}{|c|c|c|c|c|c|}
\hline \multirow[t]{2}{*}{ Group } & \multirow[t]{2}{*}{$\mathrm{n}$} & \multicolumn{4}{|c|}{ treatment effect } \\
\hline & & healed & effective & ineffective & total effective rate \\
\hline $\begin{array}{l}\text { control } \\
\text { group }\end{array}$ & $\begin{array}{l}6 \\
0\end{array}$ & 12 & 34 & 14 & $46(76.7 \%)$ \\
\hline $\begin{array}{l}\text { treatment } \\
\text { group }\end{array}$ & $\begin{array}{l}6 \\
0\end{array}$ & 23 & 33 & 4 & 56 (93.3\%) \\
\hline $\mathrm{P}$ value & & \multicolumn{4}{|c|}{$\mathrm{P}<0.05$} \\
\hline
\end{tabular}

As is shown in Table 1: In the treatment group, 23 patients were healed, 33 were effective in treatment effect, and 4 ineffective. The total effective rate of treatment in the treatment group was 93.3\%. In the control group, 12 patients recovered, 34 effective, and 14 invalid. The total effective rate of treatment in the control group was just $76.7 \%$. Thus, in terms of therapeutic effect, patients in the treatment group were significantly better than those in the control group, and $\mathrm{P}<0.05$ means discrepancy is statistically significant.

Table 2. Comparison of nursing satisfaction degree between two groups of patients

\begin{tabular}{ccccc}
\hline Group & $\mathrm{n}$ & Satisfied & Unsatisfied & $\begin{array}{c}\text { Satisfaction } \\
\text { degree }\end{array}$ \\
\hline control group & 60 & 43 & 17 & $71.6 \%$ \\
$\begin{array}{c}\text { Treatment group } \\
\text { P value }\end{array}$ & 60 & 56 & 4 & $93.3 \%$ \\
\hline
\end{tabular}

As we can see from Table 2: Of the 60 patients in the control group, 43 patients were satisfied with their care and satisfaction degree reached $71.6 \%$. In the treatment group, 56 patients were satisfied and the degree reached $93.3 \%$. It can be concluded that the satisfaction degree of the patients in the treatment group was significantly higher than that in the control group, and the $\mathrm{P}<0.05$ difference was statistically significant.

\section{Conclusions}

Hand-foot-mouth disease, a modern medical disease name, is an acute communicable disease caused by various enteroviruses. Children under four are high-risk groups. Hand-foot-mouth disease 
usually occurs in the late spring and early fall. If the child is infected, it will show a series of clinical symptoms such as minor herpes or ulcers in the oral cavity, papules in the hands and feet, and papules into herpes. At this stage, there is no potent drug for the treatment of HFMD, and HFMD has the clinical characteristics of changing rapidly, so it should be treated by TCM syndrome differentiation, which is of great significance for the rehabilitation of children ${ }^{[3]}$. From the perspective of Chinese medicine, hand-foot-and-mouth disease belongs to the category of wind and wet temperatures. From the analysis of the pathogenesis of hand-foot-and-mouth disease, the hand-foot-and-mouth disease is generally caused by the body's damp heat, and re-infected when the evil caused by the disease, when the epidemic of evil can be invaded from the children's nose and mouth, superior lung and spleen, so that lead to lung loss in children, spleen lost health movement[4].In traditional Chinese medicine, the lungs are at the source of water, the spleen is transported, and the spleen and lungs are disordered. Then the water is stopped in the body and the body is moist and hot and the skin is steamed. Therefore, herpes is present in the hands and feet and in the oral cavity. According to different emotions in clinical practice, hand-foot-and-mouth disease can be divided into three categories: wind-temperature guilty of the lungs, summer heat and flaming, and lukewarm steaming. Therefore, in the treatment of hand-foot-mouth disease in children with hand-foot-mouth disease, it is necessary to diagnose the type of hand-foot-mouth disease, so as to achieve the right medicine and rational use of drugs, so as to lay a good foundation for the rehabilitation of children [5]. The results of this study showed that 23 patients in the experimental group healed, 33 patients were effective, and 4 patients were ineffective. The total effective rate of treatment in the treatment group was 93.3\%. In the control group, 12 patients recovered, 34 patients were effective, and 14 patients were invalid. The total effective rate of treatment in the control group was only $76.7 \%$. Thus, in terms of therapeutic effect, patients in the treatment group were significantly better than those in the control group, and $\mathrm{P}<0.05$ was examined statistically significant.

In short, the use of traditional Chinese medicine method for the treatment of hand- foot-andmouth disease in children, can significantly improve the clinical treatment of children, in clinical practice is worth promoting.

\section{References}

[1] Ministry of Health of the People's Republic of China. Hand-foot-mouth disease diagnosis and treatment guidelines (2010 version) [J]. International Journal of Respiration, 2010, 30 (24): 14731474.

[2] Wang Weiwen. Clinical disease diagnosis and evaluation standard of therapeutic effect[S]. Beijing: Science and Technology Literature Publishin, 2010.8:7-8.

[3] Song Xubin, Li Fenghua, Zhu Guihua. Analysis of Hand-Foot-and-Mouth Disease Treated by Chinese and Western Medicine[J]. Journal of Community Medicine,2011,9(9):18-19.

[4] Hu Yan, Wang Mengqing. Research on characteristics and evolution law of traditional Chinese medicine syndrome in children with hand-foot-mouth disease[J]. Chinese Journal of Integrated Traditional and Western Medicine, 2011(01) :124-125.

[5] Han Dajiang. Clinical Observation of 62 Cases of Hand-foot-mouth Disease Treated with Combination of Traditional Chinese and Western Medicines[J]. Journal of Mathematical Medicine,2011, (04) :284-285. 